\title{
A Novel Flexible Framework with Automatic Feature Correspondence Optimization for Non-Rigid Registration in Radiotherapy
}

\author{
Eliana M. Vásquez Osorio B.Sc., ${ }^{*}$ Mischa S. Hoogeman Ph.D., Luiza Bondar \\ Ph.D., Peter C. Levendag M.D. Ph.D., and Ben J.M. Heijmen Ph.D. \\ Department of Radiation Oncology, Daniel den Hoed Cancer Center, \\ Erasmus Medical Center, Rotterdam, The Netherlands
}

(Dated: May 11, 2009)

Technical improvements in planning and dose delivery and in verification of patient positioning have substantially widened the therapeutic window for radiation treatment of cancer. However, changes in patient anatomy during the treatment limit the exploitation of these new techniques. To further improve radiation treatments, anatomical changes need to be modeled and accounted for. Non-rigid registration can be used for this purpose. This paper describes the design, the implementation and the validation of a new framework for non-rigid registration for radiotherapy applications. The core of this framework is an improved version of the Thin Plate Splines Robust Point Matching (TPS-RPM) algorithm. The TPS-RPM algorithm estimates a global correspondence and a transformation between the points that represent organs of interest belonging to two image sets. However, the algorithm does not allow for the inclusion of prior knowledge on the correspondence of subset of points and therefore, it can lead to inconsistent anatomical solutions. In this paper TPS-RPM was improved by employing a novel correspondence filter that supports simultaneous registration of multiple structures. The improved method allows for coherent organ registration and for the inclusion of user defined landmarks, lines and surfaces inside and outside of structures of interest. A procedure to generate control points form segmented organs is described. The framework parameters $r$ and $\lambda$, which control the number of points and the non-rigidness of the transformation respectively, were optimized for three sites with different degrees of deformation: head and neck, prostate and cervix, using two cases per site. For the head and neck 
cases, the salivary glands were manually contoured on CT-scans, for the prostate cases the prostate and the vesicles, and for the cervix cases the cervix-uterus, the bladder and the rectum. The transformation error obtained using the best set of parameters was below $1 \mathrm{~mm}$ for all the studied cases. The length of the deformation vectors were on average ( \pm 1 standard deviation) $5.8 \pm 2.5$ and $2.6 \pm 1.1 \mathrm{~mm}$ for the head and neck cases, $7.2 \pm 4.5$ and $8.6 \pm 1.9 \mathrm{~mm}$ for the prostate cases, and 19.0 \pm 11.6 and $14.5 \pm 9.3 \mathrm{~mm}$ for the cervix cases. Distinguishable anatomical features were identified for each case, and were used to validate the registration by calculating residual distances after transformation: $1.5 \pm 0.8,2.3 \pm 1.0$ and $6.3 \pm 2.9 \mathrm{~mm}$ for the head and neck, prostate and cervix sites respectively. Finally, we demonstrated how the inclusion of these anatomical features in the registration process reduced the residual distances to $0.8 \pm 0.5,0.6 \pm 0.5$ and $1.3 \pm 0.7 \mathrm{~mm}$ for the head and neck, prostate and cervix sites respectively. The inclusion of additional anatomical features produced more anatomically coherent transformations without compromising the transformation error. We concluded that the presented non-rigid registration framework is a powerful tool to simultaneously register multiple segmented organs with very different complexity.

Acknowledgments The authors thank Dr. David Teguh, Dr. Abrahim Al-Mamgani, Dr. Gerard van der Wielen and Dr. Ilse de Pree for their support and help in this study. 


\section{INTRODUCTION}

Modern radiation treatment (RT) techniques allow for planning and delivery of complex dose distributions, making an increase of the dose to target volumes and a better sparing of normal tissue possible. However, anatomical changes in patients limit the benefits of these techniques. For example, tumor shrinkage, organ deformation and internal motion introduce deviations between the planned and actually received dose, potentially causing underdosage of the target volumes and overdosage of organs at risk ${ }^{1-6}$. Thus, in order to fully exploit the advantages of modern RT techniques, anatomical changes need to be managed, among others by tracking them using non-rigid registration techniques. In non-rigid registration, two data sets (such as computed tomography (CT) scans, magnetic resonance imaging (MRI) scans, etc...) are aligned by finding a correspondence and a non-rigid transformation between elements in the two $\operatorname{sets}^{7-9}$. The computed transformation can be used to investigate and assess organ motion and deformation ${ }^{10,11}$, to determine suitable margins that encompass the whole extent of organ motion ${ }^{12,13}$, to perform automatic segmentation or delineation ${ }^{14-18}$ and to map dose distributions to a common frame of reference ${ }^{18,19}$.

One way to register the elements in the two data sets is by using the intensities in the images (CT or MRI scans) ${ }^{20-23}$. Unfortunately, the use of intensity-based non-rigid registration is limited by poor contrast between different structures or organs (especially for CT scans), the presence of artifacts in the images and the absence of overlap between structures in the reference and the deforming set. To overcome these problems, approaches using specific features in the image rather than the intensity values themselves are often used ${ }^{24-28}$. In general, these features are segmented by hand or automatically following the intensity gradients wherever possible. Most feature-based approaches rely on correspondence defined beforehand between features, either manually or automatically. The most common approach is to use single points, or landmarks, representing the same features in the two scans. Manual localization of landmarks is a tedious work and prone to errors, which in practice translates to a limited number of landmarks being identified. There are also attempts to automatically identify landmarks, for example, by automatically identifying curvatures ${ }^{25,26}$. However, this approach is not feasible in structures or organs with large deformations where no clear curvatures can be identified or are conserved.

We propose a novel approach, or framework, for non-rigid registration in which corre- 
spondence is automatically estimated and optimized. Furthermore, the framework supports multiple structures being registered simultaneously and allows the inclusion of user-defined landmarks or even features such as lines or surfaces inside and outside the structures of interest. The heart of this approach is an improved version of the non-rigid registration method originally presented by Chui et al. ${ }^{29}$, which formulates feature-based non-rigid registration as a non-rigid point matching problem. The original non-rigid registration method automatically estimates the correspondence between the points in two sets, i.e. the reference and the deforming set, and based on the estimated correspondence, calculates a non-rigid transformation function. The correspondence and the transformation between the point sets are iteratively optimized. Note that in the original method, the correspondence estimation is global, allowing all points in the sets to correspond among each other. In other words, it does not allow to include knowledge on the correspondence between subsets of points, for example subsets representing the same structure or organ. We solved this issue by introducing a filter that eliminates correspondence between points belonging to different structures, and thereby adapted the method to produce a global transformation for multiple structures. Furthermore, this filter also made possible the inclusion of selected points or lines representing anatomical features whose correspondence is known beforehand. The non-rigid transformation obtained can be applied to any point, allowing the transformation of any spatial data such as CT- images and dose distributions.

This framework has been successfully used in two recent studies. The first study quantified treatment-related local anatomical changes in a group of 10 oropharynx cancer patients ${ }^{10}$. A second study analyzed the deformation of the prostate and the seminal vesicles in prostate cancer patients to investigate the potential margin reduction allowed by on-line corrections based on intraprostatic fiducial markers ${ }^{13}$.

In the current paper, the framework for non-rigid registration is fully described, tested and anatomically validated. Within this scope, it was applied to three sites commonly treated with radiotherapy presenting different degrees of deformation: head and neck, prostate and cervix. The framework parameters $r$, which regulate the number of control points, and $\lambda$, which controls the non-rigidness of the transformation, were optimized for the three sites, using two cases per site. The accuracy of the registration was assessed by different measures: the distance between the reference and transformed surfaces as an estimate of the transformation error, and the difference between the back-transformation and forth-transformation 
as an estimate of the transformation consistency. To validate the correspondence, anatomical landmark points and lines were identified for the different cases in the reference and deforming images, and their mismatch after the registration was measured. Finally, we demonstrated the flexibility of the framework by including these landmark points and lines in the registration process, to produce more anatomical coherent transformations.

\section{METHODS AND MATERIALS}

\section{A. Patient Data}

In order to illustrate the versatility of the framework, three sites with different degrees of deformation were included in this study. A total of six example cases were analyzed, two cases per site. For each case two CT scans were used. The CT scans were pre-matched using bony anatomy in the region of interest. The structures of interest were manually delineated on axial CT scans. To obtain consistent contours in the CT scans pairs, the CT scan first contoured was used as a reference to delineate the second scan, and all contours were reviewed by a second observer.

For two oropharynx cancer patients, two CT scans were used: the scan used for planning and a repeat scan after 46 Gy, both acquired with intravenous contrast. The structures of interest were the parotid and submandibular glands. For two prostate cancer patients, two CT scans were used: the scan used for planning and a repeat scan. In each scan, the prostate and vesicles were manually delineated and merged into a single structure. The patients had four gold markers implanted in their prostate. For one cervix patient, three CT scans were acquired at different treatment days. In one CT scan the bladder was full, in another partially filled and in the last one empty. The bladder filling changes induced large deformations of the cervix-uterus. We defined two example cases: (1) partially filled vs. empty and (2) partially filled vs. full. The structures of interest were the bladder, the rectum and the cervix-uterus.

\section{B. Non Rigid Registration Framework}

The framework contains a method for generating control points from segmented structures and the non-rigid registration method, which was modified to handle multiple structures. 
Figure 1 shows a schematic of the framework. The following sections describe each of the framework components in detail.

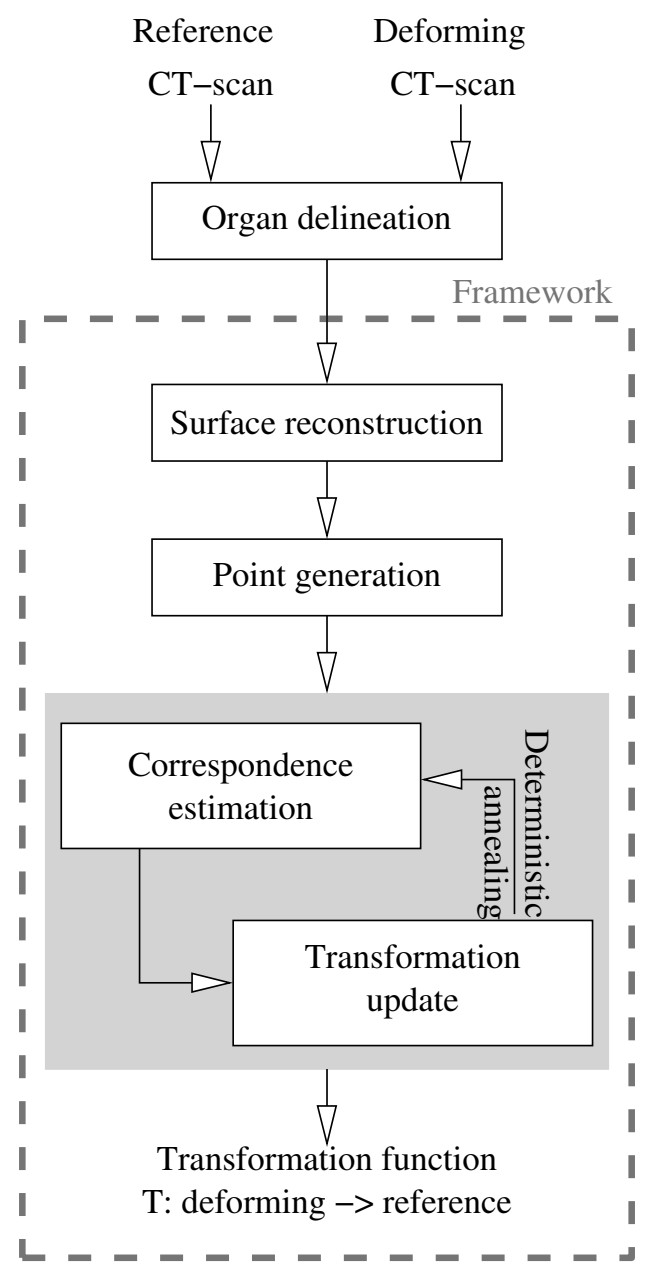

FIG. 1: Schematic of the framework. The components of the framework are enclosed by a dashed line. The heart of the framework is the non-rigid registration method presented in the gray area.

\section{Generation of control points}

Surfaces for each structure of interest were built by joining contours in consecutive slices with triangles. The points defining the triangles of the surfaces lie on consecutive planes, i.e. the axial CT slices. In the axial planes, the contour points do not follow any particular distribution. The heterogeneous point distribution in the space is not desirable in the nonrigid registration process, since the correspondence between irregularly spaced points can be problematic and error prone, and the thin plate splines work better when homogeneous 
or pseudo-homogeneous points are used ${ }^{30}$. Therefore, new points pseudo-homogeneously spread on the triangles were generated. Figure 2 illustrates the process for a simple case. First, triangles that degenerated to lines or points (two or more points are at the same xyzposition) were removed. Then, the triangles defining the surface were refined (fig. $2 \mathrm{~b}$ ). The triangles with an edge length larger than a given threshold were divided in two triangles. The edge dividing procedure was recursively repeated until all the triangles had their edge length smaller than the threshold. We used threshold values of 1.5, 1.5 and $3 \mathrm{~mm}$ for the head and neck, prostate and cervix cases, which resulted in a surface with triangles of roughly uniform size. A small threshold value is required to produce a pseudo-homogeneous point distribution. However, due to the small threshold value, the number of points exceeded the available computational resources. Therefore, we reduced the number of points using an iterative procedure. In this procedure, first a starting point is chosen to be the center of a sphere with radius $r$ (fig. 2c). All points that lie inside the sphere are replaced by the centroid of these points. The procedure is repeated with the remaining points until there are no more points left. The set of centroids is then used as control points (fig. 2d). The radius of the sphere $r$, determines the density of the resulting point set and consequently the number of control points. The described process was applied to each structure separately.

\section{Non Rigid Registration Method}

The original method, known as TPS-RPM and developed by Chui et al. ${ }^{29}$, formulates feature-based non-rigid registration as a non-rigid point matching problem. The goal is to find an optimal correspondence and transformation between two point sets, namely the reference point set, containing $n$ points, and the deforming point set, containing $k$ points. Note that $n$ and $k$ are usually different, and a one-to-one correspondence between the points is not expected.

Solving the correspondence and the transformation simultaneously is difficult, if not impossible. However both problems have an optimal solution when one of them is held fixed. Therefore the use of an iterative scheme is suitable. The process starts by estimating the correspondence between the point sets. Next, based on the estimated correspondence, the non-rigid transformation is calculated, and then applied to the deforming point set. A new iteration starts by estimating the correspondence between the newly transformed de- 


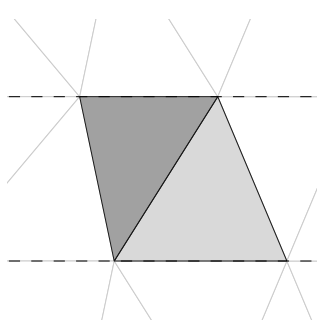

(a)

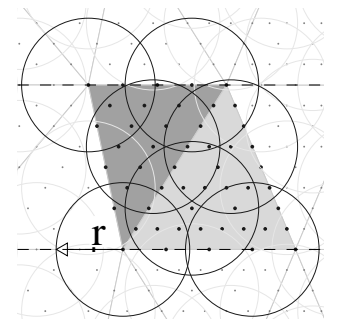

(c)

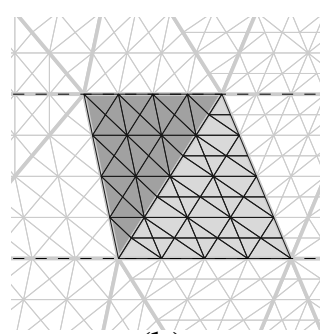

(b)

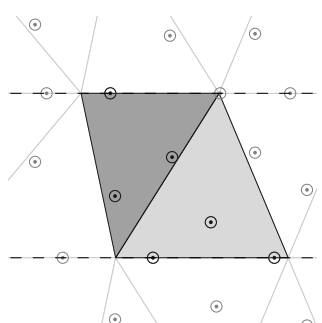

(d)

FIG. 2: Schematic showing the process to generate control points on a small part of a surface. Dashed lines represent the contours on two consecutive slices. (a) Triangles joining the original contours points. (b) Refined triangles. (c) Spheres used to group points using density radius $r$. (d) Generated control points shown as dotted circles.

forming points and the reference point set, after which the transformation is recalculated. The process is driven by a numerical technique known as deterministic annealing, which helps to avoid local minima. The annealing technique introduces a temperature variable $T$ which starts at a high value $\left(T_{\text {initial }}\right)$, and decreases over time using an annealing rate $\left(T_{k}=r_{\mathrm{a}} \times T_{k-1}\right)$, until it reaches a final temperature $T_{\text {final }}$. The common range for the annealing rate $r_{\mathrm{a}}$ is between 0.9 and 1 . We used $r_{\mathrm{a}}$ equal to 0.93 in our current implementation ${ }^{31}$.

Since the number of points in the two point sets is usually not the same, the correspondence between the different points is not binary (or one-to-one), but fuzzy. The fuzzy correspondence $\mathrm{e}^{32,33}$ allows a point in the deforming set to correspond to more than one point in the reference set and vice versa. In the current implementation a technique called softassignment was used ${ }^{34}$. The correspondence is represented by an $n \times k$ matrix $m$, where the value of $m_{i, j}, i=1 . . n$ and $j=1 . . k$, represents the degree of correspondence between two points $p_{i}$ and $q_{j}$ in the reference and deforming set respectively. The values in $m$ are restricted to be between zero and one. A value $m_{i, j}$ close to zero stands for a weak correspondence between points $p_{i}$ and $q_{j}$. A value $m_{i, j}$ close to one represents a strong correspondence 
between points $p_{i}$ and $q_{j}$. The values $m_{i, j}$ depend on the distance between the points and the current temperature value $T$ (see figure 3 ). In the first iterations the temperature $T$ is high and the correspondence $m_{i, j}$ between the points $p_{i}$ and $q_{j}$ is barely influenced by the distance between each other (see highest temperature figure 3 ). In these early stages, all points are allowed to weakly correspond to each other. With each new iteration the temperature $T$ is decreased and the distance between points starts to dominate the correspondence, to finally allow only points that are in a close proximity to correspond (lowest temperature in figure 3). We refer to Chui et al. ${ }^{29,31}$ for details about computing $m$.

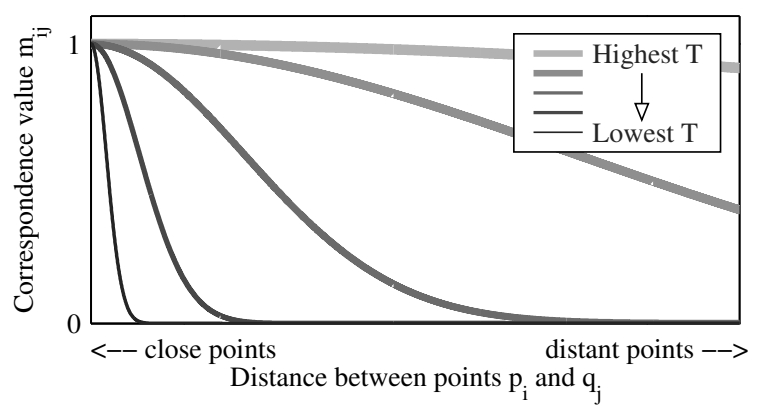

FIG. 3: Schematic showing the relation between correspondence and distance with varying temperature. The distance between two points $p_{i}$ and $q_{j}$, belonging to the reference and deforming control points set respectively, is (schematically) shown on the horizontal axis. The correspondence value between these points, $m_{i, j}$, is shown on the vertical axis. Note that the correspondence value is barely influenced by the distance between the points when the temperature is high (thick and light line), while it decreases rapidly with the distance for low temperatures.

Since no binary one-to-one correspondence is expected to exist between the two point sets, the temperature is not decreased to zero, as in conventional annealing schemes. Instead, the final temperature $T_{\text {final }}$ is equal to the mean square distance between the nearest neighbors of the deforming points ${ }^{31}$. The initial temperature $T_{\text {initial }}$ should be high enough to allow all points in the two point sets that belong to the same structure to correspond to each other. In this implementation, $T_{\text {initial }}$ was set to be the length of the diagonal of the box that bounds the largest structure.

The non-rigid transformation is modeled by thin plate splines (TPS) ${ }^{29,31}$. To determine 
the transformation function the following energy function is minimized:

$$
\min _{f} E(f)=\min _{f} \sum_{j=1}^{k}\left\|y_{j}-f\left(q_{j}\right)\right\|^{2}+\lambda T\|L f\|^{2},
$$

where $y_{j}=\sum_{i=1}^{n} m_{i, j} p_{i}, m_{i, j}$ is the correspondence matrix, $p_{i}$ are the reference points $(i=1 . . n), q_{j}$ are the deforming points $(j=1 . . k), \lambda$ is a weight parameter, $T$ is the current temperature of the deterministic annealing, and $\|L f\|^{2}$ is the smoothness measure of $f$ using the operator $L$. When the space integral of the square of the second order derivatives of $f$ is used as the operator $L$, the function that minimizes the energy function is a TPS, in the form

$$
f(x)=\phi(x) \cdot c+x \cdot d
$$

where $c$ is a $k \times 4$ matrix of warping or non-rigid coefficients, $d$ is a $4 \times 4$ affine matrix and $\phi(x)$ is a $1 \times k$ vector related to the TPS kerne ${ }^{35,36}$. Each element of $\phi(x)$ is related to the point $q_{j}$, by $\phi_{j}(x)=\left\|q_{j}-x\right\|$. The weight parameter $\lambda$ controls the degree of deformation of the transformation function, and it is annealed using the general annealing rate $\left(\lambda_{k}=r_{\mathrm{a}} \times \lambda_{k-1}\right)$. By annealing $\lambda$, it is possible to obtain a registration from a mainly affine transformation to a non-rigid one gradually. We refer to Chui et al. ${ }^{29,31}$ for further details.

\section{Correspondence Filtering}

All structures are registered simultaneously. Note that points of different structures that lie in close proximity may develop a high correspondence during the process. An incorrect correspondence would misguide the transformation leading to unacceptable results. To avoid any correspondence between points of different structures, the correspondence matrix $m$ was replaced by a filtered matrix $m_{\text {filtered }}$ defined by

$$
m_{i, j}^{\text {filtered }}= \begin{cases}m_{i, j} & \text { if } p_{i} \text { and } q_{j} \text { belong to the same structure } \\ 0 & \text { otherwise }\end{cases}
$$

In order to implement this filtering, we associated to each point an index that defined the structure it belongs to. The condition "belong to the same structure" was simply tested by checking whether index $\left(p_{i}\right)$ was the same as index $\left(q_{j}\right)$. 


\section{Forth-transformation and back-transformation}

Every registration included two transformations, a forth-transformation $(f T)$ and a backtransformation $(b T)$. The forth-transformation is found by registering the deforming set to the reference set. Likewise, the back-transformation is calculated by reversing the role of the points, and registering the reference set to the deforming set. The average length (and standard deviation) of the deformation vectors was calculated and reported. To limit the number of values reported and increase readability, the length of the vectors of the backtransformation and forth-transformation was averaged.

\section{Parameter Tuning}

Two important parameters in the non-rigid registration framework are the density radius $r$ for the generation of the point set (figure 2) and the initial value of $\lambda$ used to calculate the transformation function.

The control point density determines how much detail is included in the transformation. Few points will be enough to estimate a general pose of the structures. More points will allow fine details to be registered. However, more points also use more computational resources and time.

The $\lambda$ parameter controls the degree of deformation that a transformation can achieve. An large initial value for $\lambda$ will restrict the transformation to mostly affine, while an initial value for $\lambda$ close to zero will allow more flexible deformations. From now on, we refer to the initial value of $\lambda$ as $\lambda$.

These two parameters were tuned for the sites investigated. The distance between the transformed and the reference surfaces was used as a measure of the transformation error (see section IID for details). The error was presented as a function of the density radius $r$ and $\lambda$.

\section{Transformation error}

To assess the accuracy of the resulting transformation, we quantified the mismatch between the transformed deforming surface and its reference surface. These surfaces represent the boundary of the real structures. First, we created refined surfaces to obtain sufficient 
detail by using $2.5,2.5$, and $5 \mathrm{~mm}$ as threshold values for the head and neck, prostate and cervix cases respectively in the edge dividing procedure described in section IIB 1. Then, the transformed deforming surface was obtained by applying the computed transformation to the refined deforming surface. Next, to quantify the mismatch between the transformed deforming surface and its reference surface, the distance between these surfaces was measured. To calculate the distance between the surfaces, we computed the distance between each point $p$ in the transformed refined surface to the closest triangle in the non-refined reference surface (see figure 4). We reported the mean distance and its standard deviation. To limit the number of values reported and to increase readability, the transformation error values were averaged over the forth-transformation and back-transformation. The consistency between the forth-transformation and back-transformation was assessed separately (see next section).

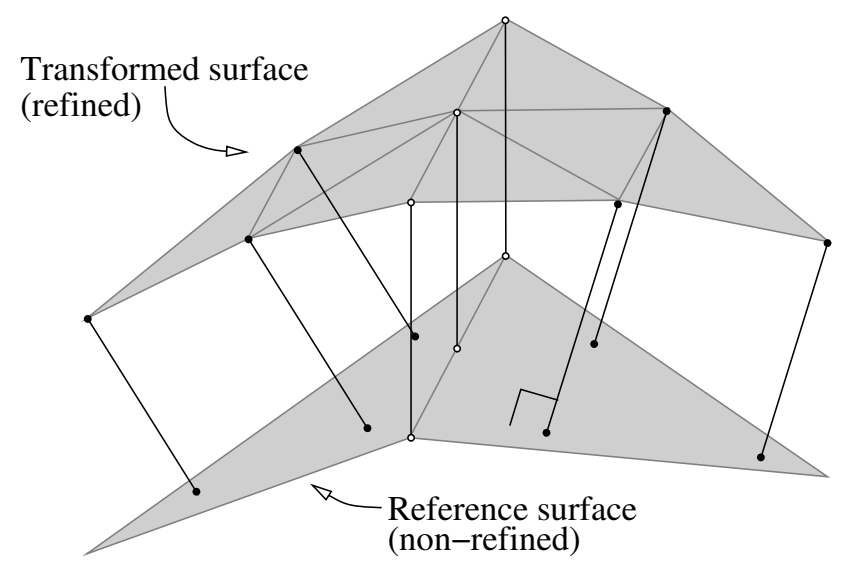

FIG. 4: Calculating the distance between a transformed deforming surface (refined) and the reference surface (non-refined). The solid points represent projected points that fall inside the closest triangle. Notice that the projection is orthogonal to the triangle plane of the non-refined surface. The empty circles represent points that fall outside the closest triangle. For these points, the distance is defined as the shortest distance to the triangle edges.

\section{E. Transformation consistency}

There is no explicit drive in the method that makes the back-transformation $(b T)$ to be the inverse of the forth-transformation $f T$, but since the input points are the same, this behavior may be expected. In practice, only the forth-transformation or the back-transformation 
is used in clinical applications, but to determine the consistency of the transformation we assessed the differences between the forth-transformations and back-transformations. In detail, a point $p$ (belonging to the surface) is transformed by $f T, p^{\prime}=f T(p)$. The transformed point $p^{\prime}$ is then transformed back by $b T\left(p^{\prime}\right)=p^{\prime \prime}$. The distance $d$ between $p$ and $p^{\prime \prime}$ for each point on the deforming and reference surface was calculated and their mean and standard deviation are reported. Note that $d$ also accounts for surface misalignments due to transformation inaccuracy (see section IID). When $f T$ and $b T$ are inverse functions of each other and the transformation error is zero, $p$ and $p^{\prime \prime}$ are mapped to the same position, and $d$ is zero. We reported the mean distance $d$ and its standard deviation.

\section{F. Correspondence of anatomical features}

Anatomical correspondence is a prerequisite for some specific applications of non-rigid registration, such as dose accumulation and quantifying anatomical changes. The transformation error and consistency do not quantify to what extent anatomical features in the deforming CT scan are transformed to the same anatomical features in the reference CT scan. To validate anatomical correspondence, anatomical features represented as points and lines were identified for the different cases. Using the transformation found with the density radius $r$ and $\lambda$ that produced the lowest error in the parameter tuning, the residual distances between the transformed deformed anatomical features and the reference features were calculated and reported.

Anatomical features were manually or automatically identified. Figure 5 shows the anatomical features on the first head and neck, prostate and cervix cases. For the head and neck cases, a line was manually drawn in the middle point of the interface between the parotid gland and the mandible and styloid process. In addition, the top and bottom of the parotid and submandibular glands were automatically found by choosing the center of mass of the 5\% highest and 5\% lowest points. For the prostate case, the start and end points of three gold markers and the tips of the vesicles were manually identified. Each gold marker was represented as a line segment joining the start and end point. The apex was automatically identified in the same way as the bottom of the parotid and submandibular glands in

the head and neck case. For the cervix cases, the midline of the uterus was automatically segmented using distance maps. The patient had four calcifications in the cervix, close to 
the cervix' surface. These calcifications, as well as the tip of the uterus and the top of the bladder were manually defined. The bottom of the bladder was defined in the same way as the bottom of the parotid and submandibular glands in the head and neck case. For the rectum no landmarks were defined, since no unmistakable points could be identified.
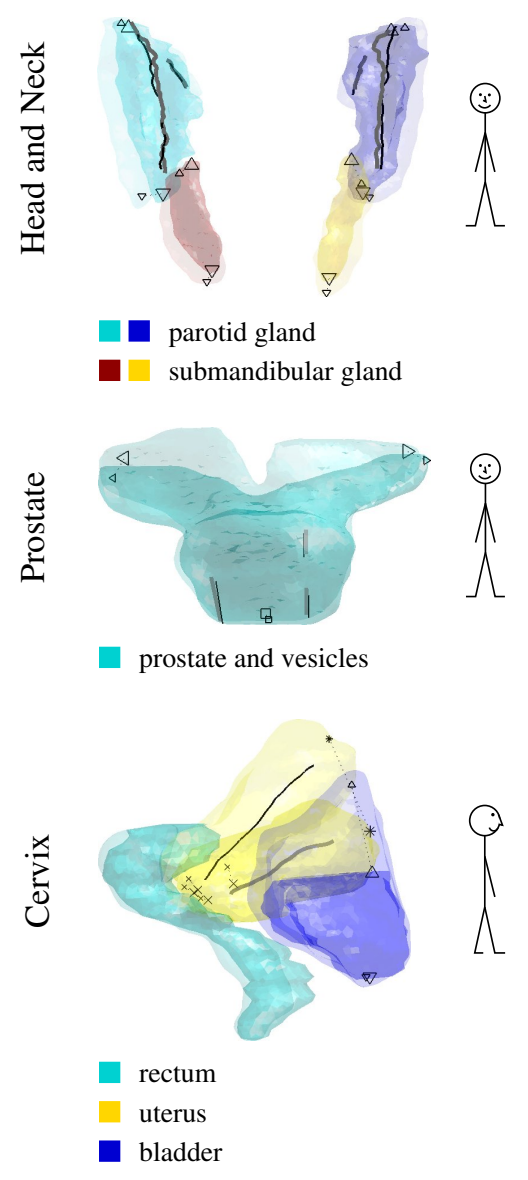

$$
\begin{aligned}
\Delta \nabla & \text { Top and bottom of organs/glands } \\
\triangleleft \triangleright & \text { Left and right vesicle tip } \\
\square & \text { Prostate apex } \\
* & \text { Uterus tip } \\
\mathrm{X} & \text { Calcifications in the cervix-uterus }
\end{aligned}
$$

FIG. 5: Anatomical features per site. Reference points and lines are shown in black, deforming points and lines are shown in gray. The images show the original overlap between deforming and reference sets: surfaces, lines and points. The lines represent the mandible - parotid gland and styloid process - parotid gland interfaces in the head and neck case, the gold markers in the prostate case and the uterus midline in the cervix case. 


\section{G. Inclusion of anatomical features in the registration}

We performed non-rigid registrations including the anatomical features identified in the previous section. The anatomical features were used to guide the non-rigid registration locally to achieve a more coherent transformation. Each anatomical feature was included in the control point set as a different structure with a unique index. Using the transformation obtained, residual distances between the transformed deformed anatomical features and the reference features were calculated and reported. The residual distances cannot be used as an independent validation for this registration as they were part of the registration process itself. Nevertheless, we reported them since the residual distances are not necessarily zero. In a non-regularized TPS transformation the residual distances would be zero, but in the current TPS implementation the degree of deformation of the transformation is regulated by $\lambda$. For example, a large value for $\lambda$ restricts the transformation to be mostly affine and would align the structures globally. This would result in a mismatch of the anatomical features for non-rigidly deforming organs. The transformation error and the transformation consistency were reported as well for the registration including anatomical features.

\section{RESULTS}

\section{A. Correspondence Filtering}

Figure 6 shows the difference between the original registration ( $m$ is not filtered) and our adapted method ( $m$ is filtered). The circles show the problematic areas that were wrongly mapped using the original method, and that were correctly mapped using our adapted method. For the head and neck case, the lower area of the parotid gland was mapped to the upper area of the submandibular gland. For the cervix case, areas in the rectum were wrongly mapped to the cervix and uterus, and the upper area of the uterus was wrongly mapped to the upper area of the bladder. By filtering $m$ during the registration, all the structures in the head and neck and cervix cases were handled correctly. 

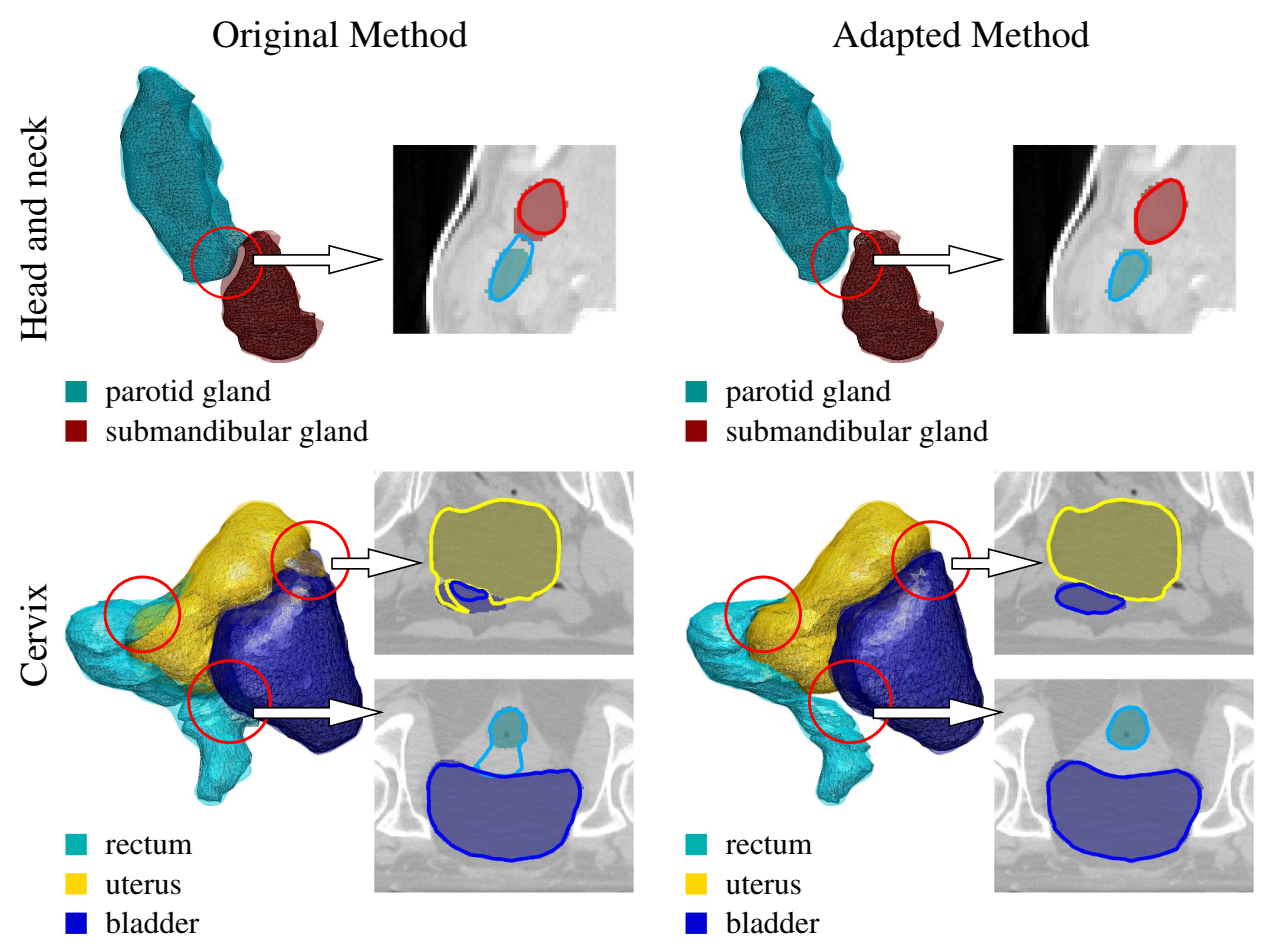

FIG. 6: Head and neck and cervix examples showing the effect of filtering the correspondence matrix. The reference and transformed surfaces are drawn in transparent and solid color respectively. Next to the surfaces, parts of axial CT images are shown, where the reference contours are presented as shadows, and the transformed contours are presented by solid lines. The first column shows the results of the original method, which does not filter the correspondence matrix $m$. The circles point out the wrong mapping between different structures. The second column shows the results of our adapted method, which filters the correspondence matrix $m$.

\section{B. Parameter Tuning}

A total of 312 registrations, that resulted in 624 back and forth transformations, were performed for parameter tuning, and the results are presented in figure 7. The transformation error, defined as the mean distance between the transformed and reference surfaces, was smaller than $1 \mathrm{~mm}$ for all the cases. The transformation error for the head and neck and prostate cases presented a shallow minimum as a function of $\lambda$. The lowest transformation error ranged between 0.4 and 0.5 (standard deviation ranged between 0.3 and 0.4 ) $\mathrm{mm}$ for $\lambda$ between 0.05 and 0.7. As expected, the transformation error reduced with decreasing density radius, producing the lowest transformation error for $r=5 \mathrm{~mm}$. For the cervix cases, 
the lowest transformation error was $0.6 \pm 0.5 \mathrm{~mm}$ (partial vs. empty) and $0.5 \pm 0.4 \mathrm{~mm}$ (partial vs. full), for $r=8 \mathrm{~mm}$ and $\lambda$ around 0.1 . The density radius $r$ and $\lambda$ parameter values that were used in the rest of the analysis are shown as red squares in figure 7.

Head and neck
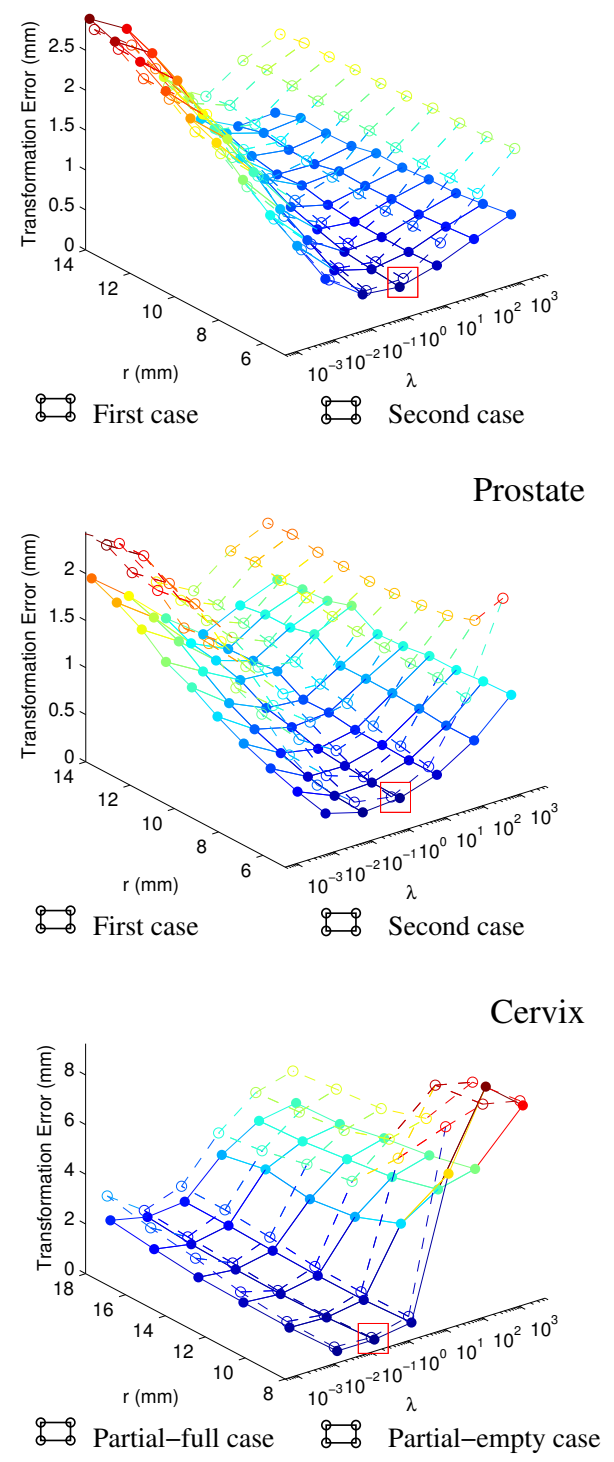

FIG. 7: Transformation error as a function of the density radius $r$ and $\lambda$ for all cases. Each graph shows the average of the back-transformation error and forth-transformation error, which was below $1 \mathrm{~mm}$. The open and solid circles show the tested parameter values and the associated transformation error. The square indicates the parameters chosen for the rest of the analysis. 


\section{Registration results}

Figure 8 shows the results of the registration of the first head and neck, prostate and cervix case. The average length of the deformation vectors was $5.8 \pm 2.5 \mathrm{~mm}$ (range 0.4 $15.7 \mathrm{~mm}$ ) and $2.6 \pm 1.1 \mathrm{~mm}$ (range $0.1-6.6 \mathrm{~mm}$ ) for the first and second head and neck case; $7.2 \pm 4.5 \mathrm{~mm}$ (range $0.9-19.9 \mathrm{~mm}$ ) and $8.6 \pm 1.9 \mathrm{~mm}$ (range $4.5-16.9 \mathrm{~mm}$ ) for the first and second prostate case, and $19.0 \pm 11.6 \mathrm{~mm}$ (range $0.6-49.7 \mathrm{~mm}$ ) and $14.5 \pm 9.3$ $\mathrm{mm}$ (range $0.2-42.3 \mathrm{~mm}$ ) for the partial vs. empty and partial vs. full case. The total number of control points was on average 1362, 975 and 2282 for the head and neck, prostate and cervix cases, and their registration took on average 30, 17 and 116 minutes respectively.

\section{Transformation consistency}

By comparing the forth-transformation $f T$ and back-transformation $b T$ for the different values of density radius $r$ and $\lambda$, we noticed that the transformation consistency worsened as $\lambda$ decreased. For large $\lambda$ values where the transformation is mostly affine, the consistency worsened again. These transformation were unacceptable, and they showed very large the transformation errors (see cervix case on figure 8, large $\lambda$, small $r$ ). In general terms, the consistency was better (smaller value) for more affine transformations than for more non-

rigid transformations. The consistency of the transformation generated with the density radius $r$ and $\lambda$ that produced the lowest error, was similar for the head and neck and prostate cases: $1.7 \pm 1.5 \mathrm{~mm}$ and $1.4 \pm 1.2 \mathrm{~mm}$ for the first and second head and neck cases and $1.6 \pm 1.4 \mathrm{~mm}$ and $1.0 \pm 0.7 \mathrm{~mm}$ for the first and second prostate cases respectively. The transformation consistency for the cervix cases was larger, $4.6 \pm 3.5 \mathrm{~mm}$ and $4 \pm 3.6$ mm for partial-empty and partial-full cases respectively.

\section{Anatomical correspondence}

Figure 9 and tables I, II and III show the registration results for the point and lines that were identified as anatomical corresponding features. The tables list the residual distances between the transformed deformed anatomical features and the reference features for the normal registration and the complemented registration. They also list the original distance 


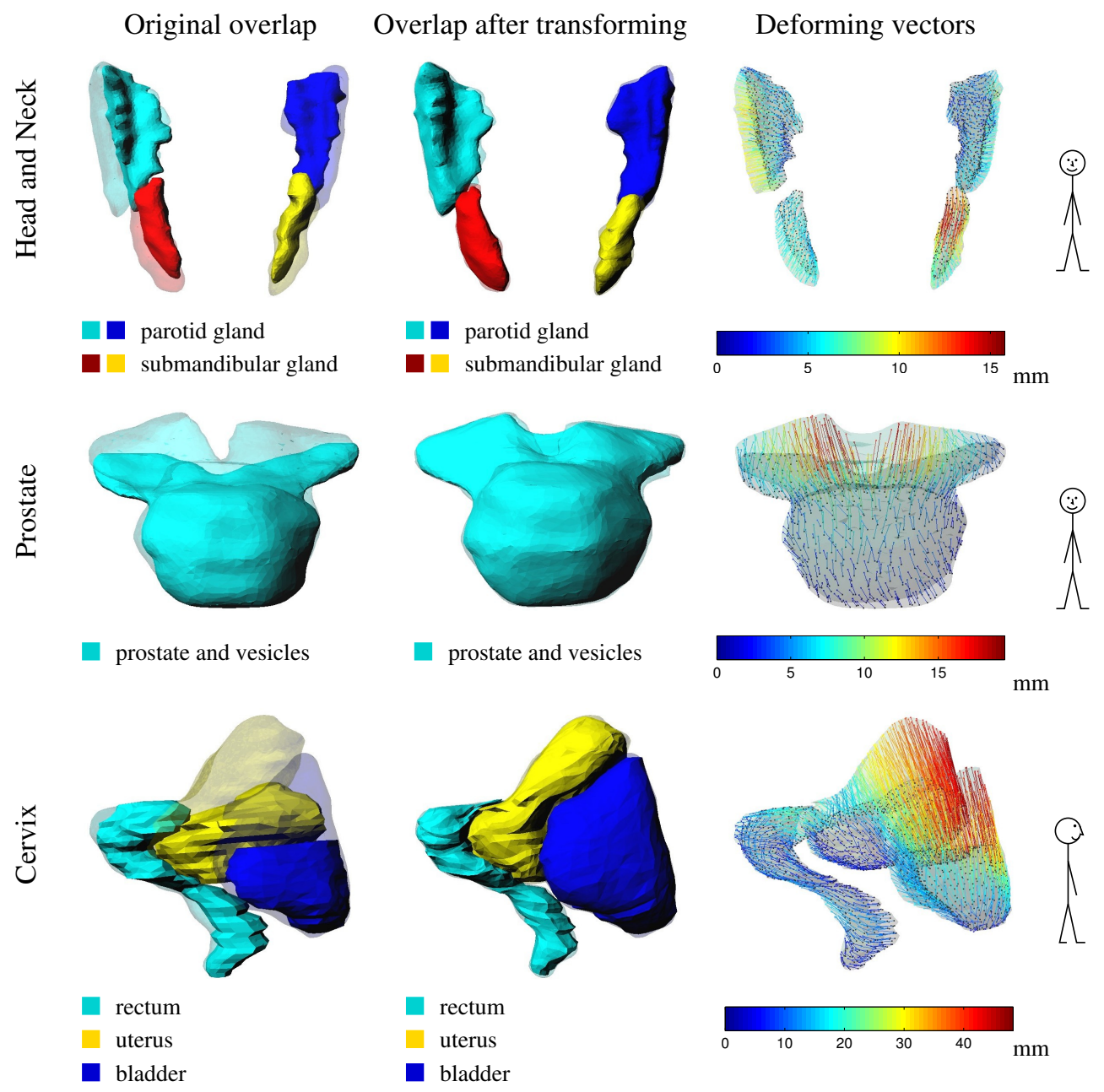

FIG. 8: Registration results. Reference surfaces are shown in transparent colors, deforming surfaces are shown in solid colors. Note that the three cases are presented in different scales. First column shows the original overlap between deforming and reference surfaces. Second column shows the overlap between transformed deforming surface and reference surface. Third column shows the deformation vectors where, for visualization purposes, the structures were separated.

between the deforming and reference features.

The residual distances between the landmarks after registration varied for the different sites. For the first head and neck case, all the residual values were below $3 \mathrm{~mm}$, except for the top point in the right submandibular $(3.8 \mathrm{~mm})$. The residual distances of the second head and neck case were all below $2 \mathrm{~mm}$. Most residual distances for the prostate cases were below $3 \mathrm{~mm}$, except for the vesicle tips (up to $3.7 \mathrm{~mm}$ ). Notice that the gold markers in the first prostate case were already very close after the rigid bone matching (around 2 


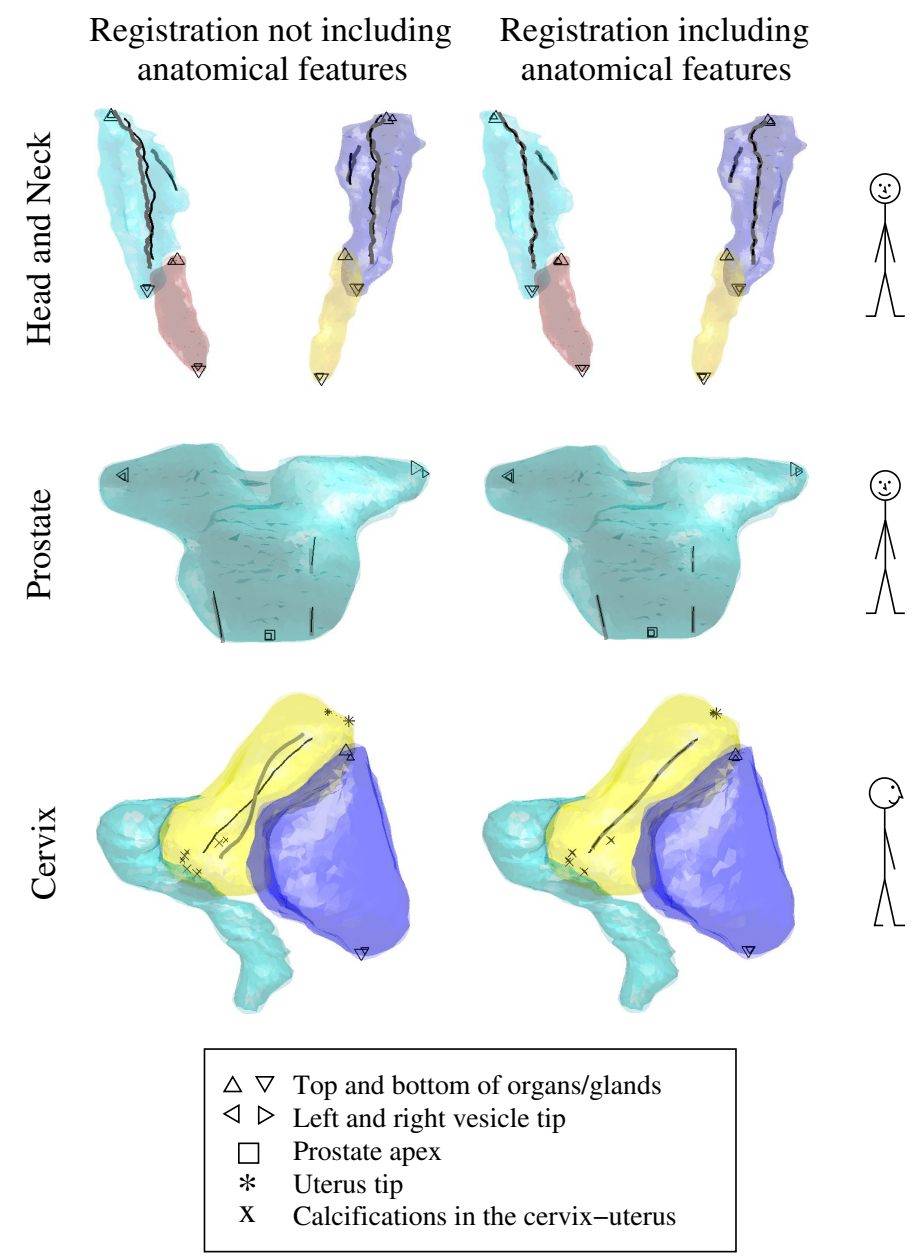

FIG. 9: Differences between the registration including and not including anatomical features. Reference points and lines are shown in black, deforming points and lines are shown in gray. The original sets are shown in figure 5. The first and second columns show the overlap between the transformed deforming set and the reference sets using the transformations resulting from not including and including anatomical features respectively. The lines represent the mandible parotid gland and styloid process - parotid gland interfaces in the first row, the gold markers in the second row and the uterus midline in the last row.

$\mathrm{mm}$ apart), opposite to the second case where the markers were relatively far from each other (around $6 \mathrm{~mm}$ ). The order of magnitude of the residual errors is larger for the cervix cases. The residual errors for the partial vs. empty bladder filling case for the cervix, varied between 2.6 and $11.2 \mathrm{~mm}$. The residual errors of the uterus tip and bladder top were the largest, above $10 \mathrm{~mm}$. The partial vs. full bladder filling case varied between 5.0 and 11 $\mathrm{mm}$. The largest error was found in one of the calcifications. 


\section{E. Inclusion of anatomical features in the registration}

As expected, all the residual distances reduced for the registrations that included the anatomical landmarks. For the first head and neck case, the residual distance was below 2 $\mathrm{mm}$, except for the top point of the right submandibular gland. For the second head and neck case, the residual distance was below $1 \mathrm{~mm}$. All the residual errors for the prostate cases reduced to below $1.5 \mathrm{~mm}$. Most of the residual errors for the cervix cases were below $2 \mathrm{~mm}$, except for the top point of the bladder in the partial vs. empty bladder filling case $(2.5 \mathrm{~mm})$ and one of the calcifications in the partial vs. full bladder filling case $(2.8 \mathrm{~mm})$.

The transformation error was not compromised by including anatomical features in the registration. The transformation error was below $1 \mathrm{~mm}$ for all cases, $0.5 \pm 0.4,0.4 \pm 0.3$ $\mathrm{mm}$ for the first and second head and neck cases, $0.4 \pm 0.3,0.4 \pm 0.3 \mathrm{~mm}$ for the first and second prostate cases and $0.6 \pm 0.5,0.5 \pm 0.4 \mathrm{~mm}$ for the cervix cases. The transformation consistency, which showed a slight improvement, was $1.4 \pm 1.3,1.0 \pm 0.8 \mathrm{~mm}$ for the first and second head and neck cases, $1.4 \pm 1.3,0.9 \pm 0.8 \mathrm{~mm}$ for the first and second prostate cases and $4.3 \pm 3.0,3.6 \pm 2.6 \mathrm{~mm}$ for the cervix cases.

\section{DISCUSSION}

The described framework estimates the correspondence as part of the registration process. This is an important difference with the currently available feature-based registration methods in radiotherapy. Brock et al. ${ }^{26}$ relied on the curvature of the structures to determine points of correspondence that were used to calculate the non-rigid registration. Schaly et al. ${ }^{27}$ generated corresponding points and applied thin-plate splines to find the non-rigid transformation, assuming that in-plane or out of plane rotations are negligible. Kaus et al. ${ }^{24}$ created corresponding points by adapting a generated surface from the reference organ to the deformed organ. However, all these methods rely on a correspondence that is known before the registration starts, and a fixed correspondence, if it is not rightly set, can be a source of error in the registration. The only attempt to dynamically adjust the corresponding points was made by Liang and $\operatorname{Yan}^{28}$. They tried to cope with the uncertainty related to the correspondence between points by iteratively changing the location of the points by minimizing a strain energy function obtained from a FEM simulation. The limitation with 
TABLE I: Distance between anatomical lines and points for the head and neck cases. Orig: Original distances after rigid bone match. AfterR: Residual distances after transformation (not including anatomical features). AfterR $+\mathrm{A}$ : Residual distances after transformation (including anatomical features).

\begin{tabular}{|c|c|c|c|c|c|c|}
\hline \multirow[b]{3}{*}{ Anatomical features } & \multicolumn{6}{|c|}{ Residual distances after transformation in $\mathrm{mm}$} \\
\hline & \multicolumn{3}{|c|}{ Case 1} & \multicolumn{3}{|c|}{ Case 2} \\
\hline & \multicolumn{3}{|c|}{ Orig AfterR AfterR+A } & \multicolumn{3}{|c|}{ Orig AfterR AfterR $+\mathrm{A}$} \\
\hline \multicolumn{7}{|l|}{ Left Parotid } \\
\hline Mandible line & 2.7 & 1.3 & 0.4 & 0.9 & 1.6 & 0.4 \\
\hline Styloid process line & 0.8 & 1.0 & 0.3 & 0.7 & 1.0 & 0.4 \\
\hline Top & 6.1 & 2.7 & 1.6 & 1.7 & 0.7 & 0.4 \\
\hline Bottom & 4.6 & 0.9 & 0.5 & 3.8 & 0.5 & 0.6 \\
\hline \multicolumn{7}{|l|}{ Right Parotid } \\
\hline Mandible line & 1.3 & 2.2 & 0.4 & 0.9 & 0.9 & 0.3 \\
\hline Styloid process line & 0.5 & 1.3 & 0.3 & 0.8 & 1.5 & 0.3 \\
\hline Top & 3.6 & 0.6 & 1.6 & 1.2 & 1.6 & 0.7 \\
\hline Bottom & 8.8 & 1.2 & 1.0 & 1.2 & 1.3 & 0.6 \\
\hline \multicolumn{7}{|l|}{ Left Submandibular } \\
\hline Top & 10.7 & 2.1 & 1.4 & 1.3 & 0.6 & 0.4 \\
\hline Bottom & 6.2 & 2.3 & 1.3 & 3.9 & 0.9 & 0.6 \\
\hline \multicolumn{7}{|l|}{ Right Submandibular } \\
\hline Top & 6.3 & 3.8 & 2.2 & 1.5 & 0.8 & 0.4 \\
\hline Bottom & 6.1 & 2.6 & 1.0 & 4.3 & 1.7 & 0.8 \\
\hline
\end{tabular}

this last approach is that it relies on one-to-one correspondence. Our framework uses the method described by Chui et $\mathrm{al}^{29}$, where the correspondence is fuzzy, allowing the registration of $n$ vs. $k$ points, and is refined progressively during the process. Our new approach of filtering the correspondence matrix, based on structure correspondence, is essential when more than one structure is being processed simultaneously (figure 6). 
TABLE II: Distance between anatomical lines and points for the prostate cases. Orig: Original distances after rigid bone match. AfterR: Residual distances after transformation (not including anatomical features). AfterR $+\mathrm{A}$ : Residual distances after transformation (including anatomical features).

\begin{tabular}{|c|c|c|c|c|c|c|}
\hline \multirow[b]{3}{*}{ Anatomical features } & \multicolumn{6}{|c|}{ Residual distances after transformation in $\mathrm{mm}$} \\
\hline & \multicolumn{3}{|c|}{ Case 1} & \multicolumn{3}{|c|}{ Case 2} \\
\hline & \multicolumn{3}{|c|}{ Orig AfterR AfterR $+\mathrm{A}$} & \multicolumn{3}{|c|}{ Orig AfterR AfterR+A } \\
\hline \multicolumn{7}{|l|}{ Prostate } \\
\hline Gold marker 1 & 2.5 & 2.7 & 0.1 & 6.7 & 1.3 & 0.1 \\
\hline Gold marker 2 & 1.3 & 1.8 & 0.2 & 6.1 & 3.0 & 0.3 \\
\hline Gold marker 3 & 1.7 & 1.3 & 0.2 & 6.1 & 1.8 & 0.1 \\
\hline Apex & 1.6 & 1.5 & 0.5 & 6.2 & 1.4 & 0.5 \\
\hline \multicolumn{7}{|l|}{ Vesicles } \\
\hline Left Tip & 6.7 & 2.0 & 0.6 & 14.9 & 3.7 & 1.5 \\
\hline Right Tip & 5.3 & 3.4 & 1.4 & 17.3 & 3.6 & 1.3 \\
\hline
\end{tabular}

Using the density radius $r$ and $\lambda$ that produced the lowest transformation error, the framework yielded reasonable transformations when only the surface of the structures was included in the process. Validating the method is difficult, since there is no golden standard to compare the results with. We measured the residual distances between corresponding anatomical features (tables I, II and III; see the 'AfterR' columns to compare the results with other studies). Similar approaches to validate non-rigid registration methods have been used in the past. Brock et $\mathrm{al}^{26}$ validated their method by tracking visible bronchial and vessel bifurcations in lung and liver, and reported an error of 1.9, 2.8 and $1.7 \mathrm{~mm}$ in the left-right (LR), anterio-posterior (AP) and inferior-superior (IS) directions respectively. Kaus et $\mathrm{al}^{24}$ measured the accuracy of their approach by tracking implanted gold markers in the prostate, and reported standard deviation of the errors of 1.6, 2.3 and $2.5 \mathrm{~mm}$ for LR, $\mathrm{AP}$ and IS directions respectively. Schaly et al. ${ }^{27}$ measured the residual distance between markers in the prostate and vesicles and reported an average registration error of $3.0 \mathrm{~mm}$. Our results are comparable (average 3D error of 2.1 and $2.5 \mathrm{~mm}$ for the first and second 
TABLE III: Distance between anatomical lines and points for the cervix cases. Orig: Original distances after rigid bone match. AfterR: Residual distances after transformation (not including anatomical features). AfterR $+\mathrm{A}$ : Residual distances after transformation (including anatomical features).

\begin{tabular}{|c|c|c|c|c|c|c|}
\hline \multirow[b]{3}{*}{ Anatomical features } & \multicolumn{6}{|c|}{ Residual distances after transformation in $\mathrm{mm}$} \\
\hline & \multicolumn{3}{|c|}{ Case 1: Partial-empty } & \multicolumn{3}{|c|}{ Case 2: Partial-full } \\
\hline & \multicolumn{3}{|c|}{ Orig AfterR AfterR+A } & \multicolumn{3}{|c|}{ Orig AfterR AfterR $+\mathrm{A}$} \\
\hline Mid-line & 24.3 & 5.7 & 0.5 & 12.0 & 5.0 & 0.4 \\
\hline Tip & 48.8 & 10.1 & 1.9 & 22.1 & 7.6 & 1.5 \\
\hline Calcification 1 & 5.4 & 3.2 & 0.9 & 4.9 & 6.5 & 1.3 \\
\hline Calcification 2 & 8.7 & 4.7 & 0.5 & 4.8 & 8.5 & 1.6 \\
\hline Calcification 3 & 7.5 & 3.2 & 0.7 & 3.4 & 11.0 & 2.8 \\
\hline Calcification 4 & 3.6 & 3.2 & 0.9 & 4.1 & 5.0 & 1.2 \\
\hline \multicolumn{7}{|l|}{ Bladder } \\
\hline Top & 45.5 & 11.2 & 2.5 & 26.3 & 8.5 & 1.9 \\
\hline Bottom & 2.1 & 2.6 & 0.5 & 3.0 & 5.1 & 1.7 \\
\hline
\end{tabular}

prostate case, not including the anatomical features in the registration). Notice that all these methods use a fixed correspondence set beforehand.

In the current study, the non-rigid registration framework was applied to three different sites. For all the studied sites, the density radius $r$ that yielded the lowest error was the smallest density radius tested (5,5 and $8 \mathrm{~mm}$ for head and neck, prostate and cervix sites respectively). A smaller density radius, would probably further reduce the transformation error, but would lead to an unacceptably high computational time. Furthermore, the achieved accuracy of $0.6 \mathrm{~mm}$ is acceptable for clinical applications. At present, the transformation obtained is applied in regions close to the structures or landmarks. The use of the transformation far from the region of interest is not yet tested nor validated. To use the current method for such an application, further research is needed.

Intraobserver variation in delineations plays an important role in the accuracy of the 
non-rigid registration. Every mistake that is made when delineating propagates in the method, since the delineated structures are the only visible information used by the framework. Including complementary information, such as internal structures clearly visible or automatically extracted features near or around the delineated structures could minimize the effect of delineation uncertainties.

The values presented in the first column ('Orig') in tables I, II and III serve as a reference of the initial mismatch between anatomical features. However, these values are, to a certain extent, arbitrary since they depend on the pre-registration used. In our study, the preregistration was rigid and based on bony anatomy in the region of interest. If a different pre-registration, e.g. based on golden markers, or no pre-registration had been used, the initial mismatch values could have been completely different.

For the cervix cases, the $\lambda$ parameter played an important role in finding the lowest transformation error. In order to reach a deformation with an acceptable transformation error, the transformation has to be able to achieve a minimum flexibility to register the organs. In this study we found an appropriate $\lambda$ by testing different values per case. However, this approach is for clinical applications not practical because of the large number of registrations that has to be performed. A possible alternative is to estimate $\lambda$ based on the amount of overlapping between the input structures, with tools such as the Jaccard index or the dice coefficient, but further experimentation and research is needed. A practical approach is to use a constant $\lambda$ value per site. Previous studies ${ }^{10,13}$ have used such an approach. Care has to be taken if the inter-patient variability in shape and deformation is large, as the use of an inappropriate $\lambda$ value could render unacceptable results.

Including complementary a-priori information in the process did not require any modification in the existing framework and resulted in a more anatomically coherent transformation. When the anatomical landmarks were included, the residual distances reduced on average to less than $1 \mathrm{~mm}$ for the head and neck and prostate cases and $1.6 \mathrm{~mm}$ for the cervix cases. The largest residual distances for the cervix cases were found for the calcifications, which were close to the surface. Notice that due to the proximity, the surface points compete in the registration with each calcification point. Consequently, the influence of a single anatomical point within close proximity to the surface is not enough to reduce the residual distances to the submillimiter range.

The registration including anatomical features was not independently validated. Error 
measures such as the transformation error and transformation consistency were calculated, and their values hardly changed. In order to independently validate this registration, additional landmarks should be identified. However, these extra landmarks could not be identified in the CT scans. To obtain additional independent landmarks other imaging modalities could be applied, e.g. MR imaging. Many of the anatomical landmarks were manually identified, such as the tip of the uterus. The identification of these anatomical landmarks depends on the observer's interpretation and on image quality. The uncertainty in the location of the landmarks is a possible source of error.

In this study we included anatomical landmarks in the form of points and lines. Points were used to represent locations for which correspondence was clear, such as the vesicle tips in the prostate cases. Lines were used to indicate the interface between bony anatomy and glands for the head and neck cases, the midline of the cervix-uterus in the cervix cases and the gold markers in the prostate cases. Lines can also be used to represent veins, arteries, foldings, air conducts, etc. Also open or partial surfaces representing structures that are not completely included in the region of interest, such as skin, bony anatomy, etc, can be included in the process by sampling their area with points, by using the procedure illustrated in figure 2. Each of these points, lines or open surfaces is considered a different organ when filtering the correspondence matrix, therefore the points that make up a line or open surface do not require one-to-one correspondence. Moreover, there is no restriction on whether the points, lines or open surfaces are on the surface, inside or outside of the structures. In this study we identified anatomical landmarks on CT scans. However, the advantages of this method can be exploited even more when images with better soft tissue contrast, such as MRI scans, are used to identify the anatomical landmarks.

The framework in its current state has a limited use for online corrections, since the computational times are large (17 minutes for around 900 points, prostate cases). However, there is room to optimize the code. On the other hand, the number of points plays a very important role in the computational time. Literature has shown that thin plate spline works best when homogeneously spread data is used ${ }^{30}$. In the current study a semihomogeneously spread set of points was generated on the surface. In this way, all parts of the surfaces are equally represented for the non-rigid registration. Nevertheless, adaptive point generation ${ }^{37,38}$, where critical areas that need more detail get denser point clouds than areas that are less critical for the registration, may yield good results in the registration and 
may also reduce the computational time when less points are needed.

\section{CONCLUSION}

The results demonstrate that our non-rigid registration framework is an effective method to simultaneously register anatomical changes of multiple organs with very different magnitudes and complexity. Moreover, a global transformation, which is locally more anatomically coherent is produced when anatomical features are added to the registration. The inclusion of additional features is fully supported by our novel flexible framework. 
* Corresponding author: Erasmus Medical Center - Daniel den Hoed Cancer Center

Department of Radiation Oncology

Groene Hilledijk 301

3075 EA Rotterdam

The Netherlands

Phone: (+31) 10-7041491

Fax: (+31) 10-7041012

; Electronic address: e.vasquezosorio@erasmusmc.nl

1 R. Mahdavi, E. Faramarzi, M. Mohammad-Zadeh, J. Ghaeammaghami, and M. V. Jabbari, “ Consequences of radiotherapy on nutritional status, dietary intake, serum zinc and copper levels in patients with gastrointestinal tract and head and neck cancer," Saudi Med J 28, 435-440 (2007).

2 W. J. de Kruijf, B. J. Heijmen, and P. C. Levendag, "Quantification of trade-off between parotid gland sparing and planning target volume underdosages in clinically node-negative head-andneck intensity-modulated radiotherapy," Int J Radiat Oncol Biol Phys 68, 136-143 (2007).

3 E. K. Hansen, M. K. Bucci, J. M. Quivey, V. Weinberg, and P. Xia, " Repeat CT imaging and replanning during the course of IMRT for head-and-neck cancer," Int J Radiat Oncol Biol Phys 64, 355-362 (2006).

4 Y. C. Kuo, T. H. Wu, T. S. Chung, K. W. Huang, K. S. Chao, W. C. Su, and J. F. Chiou, " Effect of regression of enlarged neck lymph nodes on radiation doses received by parotid glands during intensity-modulated radiotherapy for head and neck cancer," Am J Clin Oncol 29, 600-605 (2006).

5 M. Larsson, B. Hedelin, I. Johansson, and E. Athlin, "Eating problems and weight loss for patients with head and neck cancer: a chart review from diagnosis until one year after treatment," Cancer Nurs 28, 425-435 (2005).

6 J. L. Barker, A. S. Garden, K. K. Ang, J. C. O’Daniel, H. Wang, L. E. Court, W. H. Morrison, D. I. Rosenthal, K. S. Chao, S. L. Tucker, et al., " Quantification of volumetric and geometric changes occurring during fractionated radiotherapy for head-and-neck cancer using an integrated ct/linear accelerator system," Int J Radiat Oncol Biol Phys 59, 960-970 (2004). 
7 J. B. Maintz and M. A. Viergever, "A survey of medical image registration," Medical Image Analysis 2, 1-36 (1998).

8 B. Zitova and J. Flusser. "Image registration methods: a survey," Image and vision computing 21, 977-1000 (2003).

9 M. L. Kessler, "Image registration and data fusion in radiation therapy," British Journal of Radiology 79, 99-108 (2006).

10 E. M. Vásquez Osorio, M. S. Hoogeman, A. Al-Mamgani, D. N. Teguh, P. C. Levendag, and B. J. Heijmen, " Local anatomic changes in parotid and submandibular glands during radiotherapy for oropharynx cancer and correlation with dose, studied in detail with nonrigid registration," Int J Radiat Oncol Biol Phys 70, 875-882 (2008).

11 T. Guerrero, G. Zhang, T. C. Huang, and K. P. Lin, "Intrathoracic tumour motion estimation from ct imaging using the 3d optical flow method," Phys Med Biol 49, 4147-4161 (2004).

12 G. J. Meijer, J. de Klerk, K. Bzdusek, H. A. van den Berg, R. Janssen, M. R. Kaus, P. Rodrigus, and P. P. van der Toorn, "What ctv-to-ptv margins should be applied for prostate irradiation? four-dimensional quantitative assessment using model-based deformable image registration techniques," Int J Radiat Oncol Biol Phys 72, 1416-25 (2008).

13 G. J. van der Wielen, T. F. Mutanga, L. Incrocci, W. J. Kirkels, E. M. Vásquez Osorio, M. S. Hoogeman, B. J. Heijmen, and H. J. de Boer, " Deformation of the prostate and seminal vesicles relative to intraprostatic fiducial markers," Int J Radiat Oncol Biol Phys 72, 1604-1611 (2008).

14 M. J. Murphy, Z. Wei, M. Fatyga, J. Williamson, M. Anscher, T. Wallace, and E. Weiss, “ How does ct image noise affect $3 \mathrm{~d}$ deformable image registration for image-guided radiotherapy planning?," Med Phys 35, 1145-1153 (2008).

15 K. S. Chao, S. Bhide, H. Chen, J. Asper, S. Bush, G. Franklin, V. Kavadi, V. Liengswangwong, W. Gordon, A. Raben, et al., " Reduce in variation and improve efficiency of target volume delineation by a computer-assisted system using a deformable image registration approach," Int J Radiat Oncol Biol Phys 68, 1512-1521 (2007).

16 T. C. Huang, G. Zhang, T. Guerrero, G. Starkschall, K. P. Lin, and K. Forster, " Semiautomated ct segmentation using optic flow and fourier interpolation techniques," Comput Methods Programs Biomed 84, 124-134 (2006).

17 D. Ragan, G. Starkschall, T. McNutt, M. Kaus, T. Guerrero, and C. W. Stevens, "Semiautomated four-dimensional computed tomography segmentation using deformable models," Med 
Phys 32, 2254-2261 (2005).

18 J. Orban de Xivry, G. Janssens, G. Bosmans, M. De Craene, A. Dekker, J. Buijsen, A. van Baardwijk, D. De Ruysscher, B. Macq, and P. Lambin, " Tumour delineation and cumulative dose computation in radiotherapy based on deformable registration of respiratory correlated ct images of lung cancer patients," Radiother Oncol 85, 232-238 (2007).

19 G. G. Zhang, T. C. Huang, K. M. Forster, K. P. Lin, C. Stevens, E. Harris, and T. Guerrero, “ Dose mapping: validation in $4 \mathrm{~d}$ dosimetry with measurements and application in radiotherapy follow-up evaluation," Comput Methods Programs Biomed 90, 25-37 (2008).

20 J. P. Thirion, "Image matching as a diffusion process: an analogy with maxwell's demons," Med Image Anal 2, 243-260 (1998).

21 G. E. Christensen, B. Carlson, K. S. Chao, P. Yin, P. W. Grigsby, K. Nguyen, J. F. Dempsey, F. A. Lerma, K. T. Bae, M. W. Vannier, et al., " Image-based dose planning of intracavitary brachytherapy: registration of serial-imaging studies using deformable anatomic templates," Int J Radiat Oncol Biol Phys 51, 227-243 (2001).

22 M. Foskey, B. Davis, L. Goyal, S. Chang, E. Chaney, N. Strehl, S. Tomei, J. Rosenman, and S. Joshi, " Large deformation three-dimensional image registration in image-guided radiation therapy," Phys Med Biol 50, 5869-5892 (2005).

23 X. Lu, S. Zhang, H. Su, and Y. Chen, "Mutual information-based multimodal image registration using a novel joint histogram estimation," Comput Med Imaging Graph 32, 202-209 (2008).

24 M. R. Kaus, K. K. Brock, V. Pekar, L. A. Dawson, A. M. Nichol, and D. A. Jaffray, " Assessment of a model-based deformable image registration approach for radiation therapy planning," Int J Radiat Oncol Biol Phys 68, 572-580 (2007).

25 J. M. Hensel, C. Ménard, P. W. Chung, M. F. Milosevic, A. Kirilova, J. L. Moseley, M. A. Haider, and K. K. Brock, " Development of multiorgan finite element-based prostate deformation model enabling registration of endorectal coil magnetic resonance imaging for radiotherapy planning," Int J Radiat Oncol Biol Phys 68, 1522-1528 (2007).

${ }^{26}$ K. K. Brock, M. B. Sharpe, L. A. Dawson, S. M. Kim, and D. A. Jaffray, “Accuracy of finite element model-based multi-organ deformable image registration," Med Phys 32, 1647-1659 (2005).

27 B. Schaly, G. Bauman, J. Battista, and J. Van Dyk, "Validation of contour-driven thin-plate splines for tracking fraction-to-fraction changes in anatomy and radiation therapy dose map- 
ping," Phys. in Medicine and Biology 50, 459-475 (2005).

28 J. Liang and D. Yan, " Reducing uncertainties in volumetric image based deformable organ registration," Med. Phys. 30, 2116-2122 (2003).

29 H. Chui and A. Rangarajan, "A new point matching algorithm for non-rigid registration," Computer Vision and Image Understanding 89, 114-141 (2003).

30 L. Zagorchev and A. Goshtasby, "A comparative study of transformation functions for nonrigid image registration," IEEE Trans Image Process 15, 529-538 (2006).

31 H. Chui, "Non-Rigid Point Matching: Algorithms, Extensions and Applications," Ph.D. thesis, Yale University (2001).

32 K. E. Prince, "Relaxation matching techniques - a comparison," IEEE Trans Pattern Anal Mach Intell. 7, 617-623 (1985).

33 W. M. Wells III, "Statistical approaches to features-based object recognition," Int. J. Comput. Vis., 21 63-98 (1997).

34 S. Gold and A. Rangarajan. "Softmax to softassign: neural network algorithms for combinatorial optimization," Journal of Artificial Neural Networks, 2 381-399 (1996).

35 F. L. Bookstein. "Principal Warps: Thin-Plate Splines and the decomposition of deformations," IEEE Trans Pattern Anal Mach Intell., 11 567-585 (1989).

36 G. Wahba. Spline Models for Observational Data. (SIAM: Society for Industrial and Applied Mathematics, Philadelphia, 1990), p. 180.

37 N. Krahnstoever and C. Lorenz. "Computing curvature-adaptive surface triangulations of threedimensional image data," Visual Comput., 20 17-36 (2004).

38 T. Karkanis and A. J. Stewart. "Curvature-Dependent Triangulation of Implicit Surfaces," IEEE Comp Graph and App, 21 60-69 (2001). 